\title{
ON CONTRACTIONS OF SEMISIMPLE LIE GROUPS
}

\author{
BY
}

A. H. DOOLEY AND J. W. RICE

\begin{abstract}
ABSTRACr. A limiting formula is given for the representation theory of the Cartan motion group associated to a Riemannian symmetric pair $(G, K)$ in terms of the representation theory of $G$.
\end{abstract}

Introduction. Let $G$ be a connected Lie group with Lie algebra $g$, and $H$ a closed subgroup with subalgebra $\mathfrak{h}$. The coset space $G / H$ is called reductive [9, p. 389] if $\mathfrak{h}$ admits an $\operatorname{Ad}_{G}(H)$ invariant complement $m$ in $\mathfrak{g}$; i.e. a subspace $m \subset \mathfrak{g}$ such that

(i) $\mathfrak{g}=\mathfrak{h}+m$ (direct sum),

(ii) $\operatorname{Ad}_{G}(H) m \subset m$.

In this case we can form the semidirect product $m \rtimes H$ with respect to the adjoint action of $H$ on $m$. In this paper we shall restrict ourselves to the case where $G$ is semisimple with finite centre and $(G, H)$ is a Riemannian symmetric pair [10, p. 209]. Hence $H$ is contained in the fixed point set $H_{\sigma}$ of an analytic involution $\sigma$ of $G$, it contains the identity component $\left(H_{\sigma}\right)_{e}$ and $\operatorname{Ad}_{G}(H)$ is compact. Following custom, we write $K$ and $\mathfrak{f}$ rather than $H$ and $\mathfrak{h}$ in this instance. It is well known that $K$ is compact [10, p. 252] and connected if $G$ is noncompact. Furthermore $f$ is the +1 eigenspace of $d \sigma_{e}$. We make the natural choice for $m$, namely the -1 eigenspace $V$ of $d \sigma_{e}$. When $G$ is noncompact $d \sigma_{e}$ is a Cartan involution. Then $V$ is usually denoted $p$ and $\mathrm{g}=\mathfrak{f}+p$ is called a Cartan decomposition [10, p. 182]. When $G$ is compact one can choose a real form $\mathfrak{g}_{0}$ of the complexification $\mathfrak{g}_{\mathbf{c}}$ of $\mathfrak{g}$, and a Cartan decomposition $\mathrm{g}_{0}=k+p$ such that $V=i p$, i.e. $\mathrm{g}=\mathfrak{l}+i p[10, \mathrm{~V}, \S 2]$.

The semidirect product $V \rtimes K$, in the situation described above, is called the Cartan motion group associated to the pair $(G, K)$. The idea of relating the representation theories of $V \rtimes K$ and $G$ has been prevalent in the literature (cf. notably $[11,13,18])$. In particular, $V \rtimes K$ is a contraction of the Lie group $G$ in the sense of [11], and there has been some interest in understanding the relationship between the representation theories of $V \rtimes K$ and $G$ that this implies (cf. particularly the footnote on p. 343 of [17]). The aim of this paper is to give such a precise relationship. A key ingredient involved is the global counterpart of the contraction of the Lie algebra of $G$ to that of $V \rtimes K$. It is the family of smooth maps

$$
\begin{aligned}
\pi_{\lambda}: \quad & V \rtimes K \rightarrow G \\
& (v k) \rightarrow \exp _{G}(\lambda v) k .
\end{aligned}
$$

These are homomorphisms to within $O(\lambda)$ as $\lambda \rightarrow 0$.

Received by the editors August 17, 1983 and, in revised form, March 15, 1984.

1980 Mathematics Subject Classification. Primary 22E46; Secondary 43A65.

C1985 American Mathematical Society $0002-9947 / 85 \$ 1.00+\$ .25$ per page 
In $\$ 2$ we give a brief discussion of contractions of Lie groups and indicate how the Kirillov-Kostant method of orbits suggests the relationship between the representation theories of a Lie group and a contraction of it. In $\$ 3$ we give the description of the unitary irreducible representations of $V \rtimes K$ by Mackey's semidirect product analysis [19]. In particular we consider the family $\rho_{\psi, \eta}$ of irreducible representations which are called the generic irreducible representations of $V \rtimes K$. These are parametrised by a linear function $\psi$ on a maximal abelian subalgebra a of $V$, and an irreducible representation $\eta$ of $M \subset K$, where $M$ is the centraliser of $A=\exp _{G}$ a in $K$. They form a set of full Plancherel measure in the unitary dual of $V \rtimes K$ [14].

The irreducible unitary principal series representations of a noncompact semisimple Lie group $G$, with finite centre, can be given exactly the same parametrisation as the generic irreducible representations of $V \rtimes K$. We describe this in $\S 4$ and give our first main theorem, Theorem 1 , which shows how the sequence $\sigma_{n \psi, \eta}$ of principal series representations approximates $\rho_{\psi, \eta}$ in a weak sense when composed with the global contraction map $\pi_{1 / n}$.

In $\$ 5$ we prove the analogue of Theorem 1 in the compact case (Theorem 2). Once again we show how a family of irreducible representations of the compact group $G$ can be parametrised by a linear function $\psi \in a^{*}$ and an irreducible representation $\eta$ of $M$. This is a version of the Borel Weil theorem. However, the $\psi$ 's which actually give rise to representations of $G$ are not arbitrary elements of $a^{*}$ but form a cone of a lattice in it. Hence, if $\psi \in a^{*}$ is arbitrary, and we wish to approximate the irreducible representation $\rho_{\psi, \eta}$, we will not usually have available the sequence of irreducible representations of $G$ of the form $\sigma_{n \psi, \eta}$, as in the noncompact case. Instead we must choose a sequence $\psi_{1}, \psi_{2}, \ldots$ from the cone of the lattice in a ${ }^{*}$ so that $\psi_{n} / n \rightarrow \psi$. Another problem is that, in contrast with the noncompact case, $M$ and $A$ may intersect, and this forces compatibility requirements between $\psi$ and $\eta$ which affect the choice of the sequence $\psi_{1}, \psi_{2}, \ldots$. Theorem 2 shows how, with the appropriate choice of sequence, $\sigma_{\psi_{n}, \eta}$ composed with $\pi_{1 / n}$ approximates $\rho_{\psi, \eta}$ in a weak sense. The special case of this theorem where $G=S O(n+1)$ and $K=S O(n)$ was proved in [5]. An infinitesimal version for spherical functions was proved in [4].

In $\S 6$ we describe how to extend our results to the case of nongeneric irreducible representations $\rho_{\psi, \eta}$ of $V \rtimes K$. In this case $\eta$ is an irreducible representation of the stabiliser $K_{\psi}$ of $\psi$ under the coadjoint action of $K$ on $V$. We replace $M$ by $K_{\psi}$ and $A$ by $\exp a_{\psi}$, where $a_{\psi} \subset a$ is the centraliser in a of the Lie algebra ${ }_{\psi}$ of $K_{\psi}$. In the noncompact case we replace the principal series representations of $G$ by the appropriate $H$-series ( $H$.being a Cartan subgroup) and the arguments of Theorem 1 go through much the same to give complete results for the nongeneric case. If $G$ is compact, then intersection of $K_{\psi}$ with $A$ causes difficulties in repeating the arguments of Theorem 2 . This leaves the results of the compact case a little incomplete.

\section{Deformations and contractions of Lie groups.}

(2.1) Definition. Let $\mathfrak{g}$ and $\mathfrak{g}_{1}$ be two Lie algebras with the same underlying vector space $\mathscr{U}$. We say that $g_{1}$ is a deformation of $g$ if there is a set $\left(\phi_{\lambda}\right)_{\lambda \in \mathbf{R}^{+} \subseteq}$ $\mathscr{G} \mathscr{L}(\mathscr{U})$ so that $\lim _{\lambda \rightarrow 0} \phi_{\lambda}^{-1}\left[\phi_{\lambda} x, \phi_{\lambda} y\right]_{\mathfrak{g}}=[x, y]_{\mathfrak{g}_{1}}$ for all $x, y \in \mathscr{U}$. 
This definition goes back to Saletan. A number of results about deformations have been proved, for example [21, 22]. However, the definition seems too general to be useful in our context. Our main interest lies in a special case of this notion, a contraction. Before we pass to consideration of contractions let us record

(2.2) LemmA. Suppose $\mathrm{g}$ is a Lie algebra with underlying vector space $\mathscr{U}$ and $\left(\phi_{\lambda}\right)_{\lambda \in \mathbf{R}^{+}} \subseteq \mathscr{G} \mathscr{L}(\mathscr{U})$ is such that for all $x, y \in \mathscr{U},[x, y]_{1}=\lim _{\lambda \rightarrow 0} \phi_{\lambda}^{-1}\left[\phi_{\lambda} x, \phi_{\lambda} y\right]$ exists. Then []$_{1}$ is a Lie bracket on $\mathscr{U}$ and the resulting Lie algebra $\mathfrak{g}_{1}$ is a deformation of $\mathrm{g}$.

(2.3) Definition. Let $\mathfrak{g}$ be a Lie algebra with underlying vector space $\mathscr{U}$ and let $\mathfrak{f}$ be a Lie subalgebra. Let $V$ denote a subspace of $\mathscr{U}$ complementary to $\mathfrak{f}$. Thus $x \in \mathscr{U}$ can be uniquely written $x=x_{t}+x_{V}$. For $\lambda \in \mathbf{R}^{+}$, define a map $\phi_{\lambda} \in \mathscr{G} \mathscr{L}(\mathscr{U})$ by $\phi_{\lambda}(x)=x_{t}+\lambda x_{v}$.

The proof of the following lemma is computational and is left to the reader.

(2.4) Lemma. Let $x, y \in \mathscr{U}$. Then $\lim _{\lambda \rightarrow 0} \phi_{\lambda}^{-1}\left[\phi_{\lambda} x, \phi_{\lambda} y\right]$ exists and is equal to $[x, y]_{1}=\left[x_{\mathfrak{f}}, y_{\mathfrak{f}}\right]+\left(\left[x_{\mathfrak{f}}, y\right]\right)_{V}+\left(\left[x, y_{\mathfrak{f}}\right]\right)_{V}$. Thus []$_{1}$ is a Lie bracket on $\mathscr{U}$ and the resulting Lie algebra is a deformation of $\mathfrak{g}$.

A deformation arising in this way is called a contraction with respect to $\mathrm{f}$. Notice that $\mathfrak{g}_{1}$ contains $V$ as an abelian ideal and that $\mathfrak{f} \cong \mathfrak{g}_{1} / V$.

In the special case where $V$ is $\operatorname{ad}_{t}$ invariant (i.e. $[\mathfrak{f}, V] \subseteq V$ ) we have $[x, y]_{1}=$ $\left[x_{\mathrm{f}}, y_{\mathrm{f}}\right]+\left[x_{\mathrm{f}}, y_{V}\right]+\left[x_{V}, y_{\mathrm{f}}\right]$.

Contractions were introduced by Inönu and Wigner [13], who wished to study relationships between the representation theories of the two groups. Indeed, [13] contains a number of examples, for specific groups, of limiting behaviour for special functions, which are special cases of our main theorems. Other special cases have been worked out, see for example [16, 20], or Chapter 10 of [6].

We now globalize the above definitions by replacing $g$ by a Lie group $G$ and $\mathfrak{f}$ by a Lie subgroup $K$.

(2.5) Definition. Let $G$ be a Lie group and $K$ a Lie subgroup, reductive in $G$. Thus, we can write $\mathfrak{g}=\mathfrak{H} \oplus$, where the decomposition is $\operatorname{Ad}_{K}$ invariant $(\operatorname{Ad}(K) V \subseteq V)$. Let $V \rtimes K$ denote the semidirect product of $V$ by $K$ relative to this action. Define $\pi_{\lambda}: V \times K \rightarrow G$ by $\pi_{\lambda}(v k)=\exp _{G}(\lambda v) k$ for each $\lambda \in \mathbf{R}^{+}$.

(2.6) Proposition. The Lie algebra of $V \rtimes K$ is precisely $g_{1}$ of Lemma (2.4). Further, the differential of $\pi_{\lambda}$ at the identity is precisely the map $\phi_{\lambda}$ of (2.3).

Thus, we are justified in making the following definition.

(2.7) Definition. The semidirect product $V \rtimes K$ is called a contraction of $G$ with respect to $K$, and the family $\left(\pi_{\lambda}\right)_{\lambda \in \mathbf{R}^{+}}$of maps $V \rtimes K \rightarrow G$ is called the family of contraction maps.

Our canonical example of a contraction is that outlined in the introduction, where $(G, K)$ is a Riemannian symmetric pair and the contraction of $G$ with respect to $K$ is the associated Cartan motion group. It seems, however, that some of the methods 
used here might extend to further examples, for instance where $K$ is noncompact. Indeed, there are infinitesimal results in this setting (cf. [11]).

The Kirillov-Kostant method of orbits [2] gives further evidence of a definite relationship in general between the irreducible representations of a Lie group and a contraction of it; indeed the method of orbits inspired us to the theorems of this paper. One considers the orbits of a Lie group $G$ under the coadjoint action on the dual $\mathrm{g}^{*}$ of its Lie algebra. If $G_{\psi}$ is the stabiliser of $\psi \in \mathrm{g}^{*}$, one calls the orbit through $\psi$ integral if there is a character $\chi$ of $G_{\psi}$ such that $d \chi_{e}=2 \pi i \psi$ on $\mathrm{g}_{\psi}$. One then forms the $G$-module induced from $\chi$. The set of such $G$-modules so obtained (from various possible choices of $\chi$ ) depends only on the coadjoint orbit through $\psi$ and not $\psi$ itself. From further geometrical data, namely a choice of polarisation on the orbit $[2,7]$, one can produce irreducible representations of $G$.

The simplest example will now serve to make our point. Let $G=S O(3)$ and $K$ be the subgroup leaving, say, the $z$-axis fixed. The Lie algebra $5 o(3)$ is identified with $\mathbf{R}^{3}$ and the family

$$
\begin{aligned}
\phi_{\lambda}: & \mathbf{R}^{3} \rightarrow \mathbf{R}^{3} \\
& (x, y, z) \mapsto(\lambda x, \lambda y, z)
\end{aligned}
$$

defines a contraction of $\mathfrak{g} o(3)$ with respect to $\mathfrak{f}$. The contracted group is the Euclidean motion group of $\mathbf{R}^{2}$, i.e. $M_{2}=\mathbf{R}^{2} \rtimes S O(2)$. Each circular cylinder with axis the $z$-axis is an integral orbit of the coadjoint action of $M_{2}$ on $\mathbf{m}_{2}^{*} \equiv \mathbf{R}^{3}$. Each sphere of integer radius with centre $0 \in \mathbf{R}^{3}$ is an integral coadjoint orbit of $S O(3)$ on $\Xi o(3)^{*}=\mathbf{R}^{3}$. In these cases, despite the possible choices of characters and polarisations, each orbit produces a unique irreducible unitary representation of the group concerned. Let $\rho_{R}$ be the representation of $M(2)$ deriving from the cylinder of radius $R>0$, and $\sigma_{n}$ the representation of $S O(3)$ deriving from the sphere of radius $n \in \mathbf{N}$. If we let $\lambda_{n}=R / n$, then the image of the sphere of radius $n$ under $\phi_{\lambda_{n}}$ is a cigar shape (for $\lambda_{n}<1$ ) of height $n$, which is tangent to the cylinder of radius $R$ along their intersection with the $x-y$ plane. The sequence of images for $n=1,2, \ldots$ is a sequence of cigar shapes which converges, in a sense, to the cylinder. Loosely speaking one may say that, upon following through the Kirillov-Kostant construction, the associated representations exhibit a similar convergence; that the sequence $\sigma_{n}$, distorted by the values $\pi_{\lambda_{n}}$ of the global contraction, converge in a sense to $\rho_{R}$.

In general, if $\bar{G}$ is a contraction of a Lie group $G$, then to each integral coadjoint orbit of $\bar{G}$ we expect to find a sequence of integral coadjoint orbits of $G$ converging to it under the adjoint of the contracting family, as in the example above. We would further expect to produce a convergence of associated irreducible representations. Theorems 1 and 2 of this paper realize these ideas in the case of the contraction of a Riemannian symmetric pair to its associated Cartan motion group. It has proved more convenient in this case to expound the representation theory in the more conventional form, rather than to persist with the coadjoint orbit description.

3. Irreducible representations of Cartan motion groups. Let $(G, K)$ be a Riemannian symmetric pair. We summarise the description of the unitary dual of the associated Cartan motion group $V \rtimes K$ via Mackey's normal subgroup analysis [18, 
19]. We consider $V \rtimes K$ as the group generated by $V$ and $K$ subject to the relations

$$
k v k^{-1}=\operatorname{Ad}(k) v \quad \forall v \in V, k \in K .
$$

Any character $\chi$ of the vector group $V$ can be uniquely expressed as $e^{i \psi}$ for some $\psi \in V^{*}$, and this identifies the character group $\hat{V}$ with the dual space $V^{*}$. For Mackey's analysis one must consider the action of $K$ on $\hat{V}$ defined by

$$
k \cdot \chi(v) \equiv \chi\left(k^{-1} v k\right)=e^{i \psi}\left(\operatorname{Ad}\left(k^{-1} v\right)\right)
$$

which clearly coincides with the coadjoint action of $K$ on $V^{*}$. For any $\psi \in V^{*}$ let $K_{\psi}$ be the stabiliser of $\psi$ under the coadjoint action of $K$, and let $\eta$ be an irreducible unitary representation of $K_{\psi}$ on some Hilbert space $H_{\eta}$. Then $e^{i \psi} \otimes \eta$ is an irreducible representation of the subgroup $V \rtimes K_{\psi}$ on $H_{\eta}$, namely

$$
e^{i \psi} \otimes \eta(v m) \equiv e^{i \psi(v)} n(m) \quad \forall v \in V, m \in K_{\psi} .
$$

The representation of $V \rtimes K$ induced from $e^{i \psi} \otimes \eta$ gives rise to an irreducible unitary representation which we denote by $\rho_{\psi, \eta}$. Every irreducible unitary representation of $V \rtimes K$ occurs in this way [19, Theorems $A$ and B, pp. 42, 43].

By its definition $\rho_{\psi, \eta}$ acts on a space of functions $f: V \rtimes K \rightarrow H_{\eta}$ satisfying

$$
e^{i \psi(v)} \eta(m) f(g v m)=f(g) \quad \forall v \in V, m \in K_{\psi}, g \in V \rtimes K .
$$

Consequently,

$$
f(v k)=f\left(k \operatorname{Ad}\left(k^{-1}\right) v\right)=e^{-i \psi\left(\operatorname{Ad}\left(k^{-1}\right) v\right)} \cdot f(k)
$$

and any such $f$ is uniquely determined by its restriction to $K$. For the representation of $\rho_{\psi, \eta}$ one considers only those functions whose restrictions to $K$ lie in $L^{2}\left(K, H_{\eta}\right)$. These restrictions comprise the closed subspace $H_{\psi, \eta}$ of $L^{2}\left(K, H_{\eta}\right)$ whose functions $f$ satisfy

$$
\eta(m) f(k m)=f(k) \quad \forall m \in K_{\psi}, k \in K
$$

In this way the representation space of $\rho_{\psi, \eta}$ inherits a Hilbert space structure on which $\rho_{\psi, \eta}$ acts unitarily. Note that

$$
\begin{aligned}
\rho_{\psi, \eta}(v k) f\left(k_{0}\right) & \equiv f\left(k^{-1}(-v) k_{0}\right)=f\left(k^{-1} k_{0} \operatorname{Ad}\left(k_{0}^{-1}\right)(-v)\right) \\
& =e^{i \psi\left(\operatorname{Ad}\left(k_{0}^{-1}\right) v\right)} f\left(k^{-1} k_{0}\right)
\end{aligned}
$$

which allows one to define $\rho_{\psi, \eta}$ directly on $\mathscr{H}_{\psi, \eta}$.

In order for two irreducible representations $\rho_{\psi_{1}, \eta_{1}}$ and $\rho_{\psi_{2}, \eta_{2}}$ to be unitarily equivalent, it is necessary and sufficient that $\psi_{1}$ and $\psi_{2}$ lie in the same coadjoint orbit of $K$, and that $\eta_{1}$ and $\eta_{2}$ be unitarily equivalent (note that $K_{\psi_{1}}$ and $K_{\psi_{2}}$ are conjugate subgroups in this case). Because $K$ is compact we may endow $V$ with an $\operatorname{Ad}(K)$-invariant inner product $\langle$,$\rangle (for example, the Killing form restricted to V$ ) by which we identify $V$ with $V^{*}$, and the adjoint with the coadjoint action of $K$. Let $a \subset V$ be a maximal abelian subalgebra of $V$. Every adjoint orbit of $K$ in $V$ intersects a [10, p. 247]. Hence every irreducible unitary representation of $V \rtimes K$ has the form $\rho_{\psi, \eta}$, where $\psi$ has the form $v \mapsto\langle H, v\rangle$ for some $H \in$ a. Such a $\psi$ can be regarded as 
an element of $a^{*}$ which has been extended uniquely to $V$ by making it 0 on the orthogonal complement of $a$. In this sense the unitary dual of $V \rtimes K$ is described by the representations $\rho_{\psi, \eta}$ with $\psi \in \mathfrak{a}^{*}$.

For $\psi \in V^{*}$ of the form $v \mapsto\langle H, v\rangle, K_{\psi}$ is the same as the stabiliser of $H$ under the adjoint action of $K$. Let $A \equiv \exp _{G}$ a and $M$ be the centraliser of $\mathfrak{a}$ in $K$. Except on certain hyperplanes, the root hyperplanes, the stabiliser of $H \in \mathfrak{a}$ is $M[10, \mathrm{p}$. 263]. We call such $H$ 's, the corresponding $\psi$ 's and associated irreducible representations $\rho_{\psi, \eta}$ generic. Because the nongeneric elements of a have Lebesgue measure zero, one can show that the nongeneric irreducible representations in the unitary dual of $V \rtimes K$ have Plancherel measure zero [14]. In what follows we shall be chiefly concerned with the generic case.

4. The noncompact case. Let $(G, K)$ be a Riemannian symmetric pair of the noncompact type. Let $a \subset V$ be a maximal abelian subalgebra of $V, A=\exp _{G}$ a, $G=K A N$ an Iwasawa decomposition, and $M$ the centraliser of $A$ in $K$ [10, p. 270]. If $\gamma \in a^{*} \otimes \mathbf{C}$ and $\eta$ is an irreducible unitary representation of $M$, we define the representation $e^{\gamma} \otimes \eta$ of $M A N$ by

$$
e^{\gamma} \otimes \eta: \text { man } \mapsto e^{\gamma(\log a)} \eta(m) \quad \forall m \in M, a \in A, n \in N,
$$

where $\log a \in \mathfrak{a}$ is defined by $a=\exp _{G}(\log a)$. A principal series representation of $G$ is one induced from a representation $e^{\gamma} \otimes \eta$ of $M A N$. As such, it is realised on a space of functions $F: G \rightarrow H_{\eta}$, where $H_{\eta}$ is the representation space of $\eta$, and satisfying

$$
e^{\gamma(\log a)} \eta(m) f(\text { gman })=f(g) \quad \forall g \in G, \text { man } \in M A N .
$$

By the Iwasawa decomposition, such functions are clearly determined by their restrictions to $K$.

In general, principal series representations do not give rise to unitary representations. Where they do their representation spaces consist of the functions satisfying the above condition, and whose restrictions to $K$ lie in $L^{2}\left(K, H_{\eta}\right)$. These restrictions comprise the subspace of $L^{2}\left(K, H_{\eta}\right)$ whose functions $f$ satisfy

$$
\eta(m) f(k m)=f(k) \quad \forall k \in K, m \in M .
$$

The representation space is thus endowed with a Hilbert space structure. A principal series representation acts unitarily on this Hilbert space if and only if $\gamma=\mu+i \psi$, where $\psi \in a^{*}$ and $\mu \in a^{*}$ is a particular linear map; its form will not concern us, but for the sake of completeness we note that [23] $\mu=\sum_{\alpha \in P^{+}} m_{\alpha} \alpha$, where $P^{+}$is the set of positive restricted roots and $m_{\alpha}$ is the multiplicity of $\alpha\left[10\right.$, p. 264]. By $\sigma_{\psi, \eta}$ we denote the unitary principal series representation of $G$ induced from $e^{(\mu+i \psi)} \otimes \eta$, and by $H_{\psi, \eta}$ its representation space.

To any generic irreducible unitary representation of $V \rtimes K, \rho_{\psi, \eta}$, we can associate the sequence $\left\{\sigma_{n \psi, \eta}\right\}_{1}^{\infty}$ of unitary principal series representations of $G$. We shall show how this sequence approximates $\rho_{\psi, \eta}$. For this we will need the global contraction

$$
\begin{aligned}
\pi_{\lambda}: & V \rtimes K \rightarrow G \\
& v k \rightarrow \exp _{G}(\lambda v) k
\end{aligned}
$$


and the function

$$
\begin{aligned}
s_{\psi}: \quad & G \rightarrow \mathbf{C} \\
k a n & \rightarrow e^{-i \psi(\log a)} .
\end{aligned}
$$

Obviously $s_{\psi}$ is $K$ invariant and has the value 1 on $K$. Furthermore, if $f \in H_{n \psi, \eta}$ then $s_{\psi} f \in H_{(n+1) \psi, \eta}$, and $s_{\psi} f$ has the same restriction to $K$ as $f$. Thus multiplication by $s_{\psi}$ identifies $H_{n \psi, \eta}$ with $H_{(n+1) \psi, \eta}$ in a way that identifies functions with the same restriction to $K$.

(4.1) Lemma. If $H \in \mathfrak{a}$ and $X \in \mathfrak{f}+\mathfrak{n}$, then

$$
s_{\psi}\left(\exp _{G}(H+t X)\right)=s_{\psi}\left(\exp _{G} H\right)+O\left(t^{2}\right) \text {. }
$$

Proof. Write $X=X_{1}+X_{2}$, where $X_{1} \in \mathfrak{f}$ and $X_{2} \in \mathfrak{n}$. Both $t \rightarrow \exp _{G}(H+t X)$ and $t \rightarrow \exp _{G} t X_{1} \cdot \exp _{G} H \cdot \exp _{G} t X_{2}$ have the same tangent vector at $t=0$, so that $t \rightarrow s_{\psi}\left(\exp _{G}(H+t X)\right)$ and $t \rightarrow s_{\psi}\left(\exp _{G} t X_{1} \cdot \exp _{G} H \cdot \exp _{G} t X_{2}\right)$ have the same derivative at $t=0$. By the definition of $s_{\psi}$ the second function is constant and so has derivative zero. The lemma follows by Taylor's theorem.

(4.2) Corollary. Let $\psi \in \mathfrak{a}^{*}$ and extend it to $V$ via the Killing form, i.e. represent $\psi(-)=\langle H,-\rangle$ for $H \in \mathfrak{a}$. Then $s_{\psi}^{n}\left(\exp _{G}-v / n\right)=e^{i \psi(v)}+O(1 / n)$.

Proof. Let $v=v_{k}+v_{a}+v_{n}$, where $v_{k} \in \mathfrak{f}, v_{a} \in \mathfrak{a}, v_{n} \in \mathfrak{n}$. By the lemma

$$
s_{\psi}\left(\exp _{G}-v / n\right)=s_{\psi}\left(\exp _{G}-v_{a} / n\right)+O\left(1 / n^{2}\right) \text {. }
$$

By the definition of $s_{\psi}$

$$
s_{\psi}\left(\exp _{G}-v_{a} / n\right)=e^{+i \psi}\left(v_{a} / n\right)
$$

Hence

$$
s_{\psi}^{n}\left(\exp _{G}-v_{a} / n\right)=e^{i \psi\left(v_{a}\right)}+O(1 / n) .
$$

Since $\mathfrak{f}, a$ and $n$ are orthogonal under the Killing form, $\psi\left(v_{a}\right)=\psi(v)$.

(4.3) THEOREM 1. With the notation above, define

$$
\bar{\sigma}_{n \psi, \eta}(v k): f \mapsto \sigma_{n \psi, \eta}\left[\pi_{1 / n}(v k)\right]\left(s_{\psi}^{n} f\right)
$$

$\forall v k \in V \rtimes K$ and $f \in H_{0, \eta}$. Then

$$
\operatorname{Lim}_{n \rightarrow \infty} \sigma_{n \psi, \eta}(v k)(f)_{\mid k}=\rho_{\psi, \eta}(v k)\left(f_{\left.\right|_{K}}\right) .
$$

The limit is in the sense of $L^{2}\left(K, H_{\eta}\right)$.

Proof. For $f \in H_{0, \eta}, v k \in V \rtimes K$ and $k_{0} \in K$ we have

$$
\begin{aligned}
\bar{\sigma}_{n \psi, \eta}(v k) f\left(k_{0}\right) & \equiv \sigma_{n \psi, \eta}\left[\pi_{1 / n}(v k)\right]\left(s_{\psi}^{n} f\right)\left(k_{0}\right) \\
& =s_{\psi}^{n} f\left(\left(\exp _{G}\left(\frac{v}{n}\right) k\right)^{-1} k_{0}\right) \\
& =s_{\psi}^{n}\left(k^{-1} k_{0} \exp _{G}-\operatorname{Ad}\left(k_{0}^{-1}\right) \frac{v}{n}\right) f\left(k^{-1} k_{0} \exp _{G}-\operatorname{Ad}\left(k_{0}^{-1}\right) \frac{v}{n}\right) .
\end{aligned}
$$


By the $K$-invariance of $s_{\psi}$ and the corollary above

$$
s_{\psi}^{n}\left(k^{-1} k_{0} \exp _{G}-\operatorname{Ad}\left(k_{0}^{-1}\right) \frac{v}{n}\right)=e^{i \psi\left(\operatorname{Ad}\left(k_{0}^{-1}\right) v\right)}+O\left(\frac{1}{n}\right) .
$$

Hence

$[*]$

$$
\begin{aligned}
\bar{\sigma}_{n \psi, \eta}(v k) f\left(k_{0}\right)= & e^{i \psi\left(\operatorname{Ad}\left(k_{0}^{-1}\right) v\right)} f\left(k^{-1} k_{0}\right) \\
& +e^{i \psi\left(\operatorname{Ad}\left(k_{0}^{-1}\right) v\right)}\left[f\left(k^{-1} \exp _{G}\left(-\frac{v}{n}\right) k_{0}\right)-f\left(k^{-1} k_{0}\right)\right]+O\left(\frac{1}{n}\right)
\end{aligned}
$$

Since

$$
\rho_{\psi, \eta}(v k) f\left(k_{0}\right)=e^{i \psi\left(\operatorname{Ad}\left(k_{0}^{-1}\right) v\right)} f\left(k^{-1} k_{0}\right)
$$

we obtain

$$
\left\|\overline{\boldsymbol{\sigma}}_{n \psi, \eta}(v k)(f)-\rho_{\psi, \eta}(v k)\left(f_{\left.\right|_{K}}\right)\right\|=\left\|\boldsymbol{\sigma}_{0, \eta}\left(\exp _{G}\left(\frac{v}{n}\right)\right) f_{\left.\right|_{K}}-f\right\|+O\left(\frac{1}{n}\right),
$$

where \|\| is the norm on $L^{2}\left(K, H_{\eta}\right)$. The theorem follows by the strong continuity of $\sigma_{0, \eta}$.

(4.4) Corollary. If $f \in H_{0, \eta}$ is a smooth function, then

$$
\left\|\bar{\sigma}_{n \psi, \eta}(v k)(f)_{\mid K}-\rho_{\psi, \eta}(v k)\left(f_{\left.\right|_{K}}\right)\right\|=O(1 / n)
$$

with the estimate uniform on compact subsets of $V \rtimes K$.

Proof. For such an $f$

$$
f\left(k^{-1} \exp _{G} \frac{-v}{n} k_{0}\right)=f\left(k^{-1} k_{0}\right)+O\left(\frac{\|v\|}{n}\right)
$$

by the mean value theorem, and the smoothness of $f$ guarantees uniformity of the estimate on $K$. Hence equation [*] in Theorem 1 can be replaced by

$$
\bar{\sigma}_{n \psi, \eta}(v k) f\left(k_{0}\right)=e^{i \psi\left(\operatorname{Ad}\left(k_{0}^{-1}\right) v\right)} f\left(k^{-1} k_{0}\right)+O(1 / n)
$$

and the corollary follows.

5. The compact case. We shall describe the irreducible representations of a compact connected Lie group by a form of the Borel-Weil-Bott theorem [25, Chapter 6]. Let $\mathfrak{g}$ be the Lie algebra of $G$ and $\mathfrak{g}_{\mathbf{c}} \equiv \mathfrak{g} \otimes \mathbf{C}$ its complexification. We let $\mathfrak{g}$ and $g_{\mathrm{c}}$ act on $C^{\infty}(G)$ as left invariant vector fields; i.e. for $X \in \mathfrak{g}$ and $f \in C^{\infty}(G)$

$$
X f(g)=\frac{d}{d s} f(g \exp s X)_{\mid s=0} \quad \forall g \in G
$$

and for $X \in g_{\text {c }}$

$$
X f=X_{1} f+i X_{2} f \quad \text { where } X=X_{1}+i X_{2}, X_{1}, X_{2} \in g .
$$

Fix a maximal torus $T$ with Lie algebra t. The adjoint action of $T$ on $g_{c}$ decomposes into a sum of characters $e^{i \alpha}, \alpha \in \mathrm{t}^{*}$. The $\alpha$ 's which occur are called the roots of $\mathfrak{g}_{\mathrm{c}}$ with respect to $T$, and the one-dimensional representation space $g_{\alpha} \subset g_{c}$ of $e^{i \alpha}$ is 
called the root space of $\alpha$. The characters occur in pairs $e^{i \alpha}, e^{-i \alpha}$, and by suitably ordering $\mathrm{t}[12$, p. 47], one chooses one out of each pair $\alpha,-\alpha$ of roots as being the set $\Phi^{+}$of positive roots. Let $\mathfrak{n}$ be the sum of the positive root spaces, i.e.

$$
\mathfrak{n}=\sum_{\alpha \in \Phi^{+}} \mathfrak{g}_{\alpha} .
$$

For any character $\chi$ of $T$, the subspace of $C^{\infty}(G)$ satisfying

$$
\begin{gathered}
\chi(t) f(g t)=f(g) \quad \forall t \in T, g \in G, \\
X f=0 \quad \forall X \in \mathfrak{n},
\end{gathered}
$$

where $f \in C^{\infty}(G)$, is invariant under the left regular representation. The restriction of it to this subspace is called the representation of $G$ induced holomorphically from $\chi$, and we denote it $\mathscr{H}_{\chi}$. Condition (iv) is the usual induced representation condition, and $(v)$ is called the holomorphy condition.

The Borel-Weil-Bott theorem asserts that every irreducible representation of $G$ has the form $\mathscr{H}_{\chi}$ for some unique $\chi$, and that in general $\mathscr{H}_{\chi}$ is either irreducible or zero. Furthermore, let us use the Killing form ( , ) of $g$ restricted to $t$ to represent each root $\alpha$ in the form $\left(t_{\alpha},-\right)$ for some $\mathrm{t}_{\alpha} \in \mathrm{t}$. $\mathscr{H}_{\chi}$ is nonzero if and only if

$$
d \chi_{e}\left(t_{\alpha}\right) \leq 0 \quad \forall \alpha \in \Phi^{+} .
$$

We shall now convert the Borel-Weil-Bott description of the irreducible representations of a compact connected semisimple Lie group $G$ into a form resembling the principal series of a noncompact such group. Let $(G, K)$ be a Riemannian symmetric pair of compact type and, as before, let a $\subset V$ be a maximal abelian subalgebra of $V, A=\exp _{G}$ a and $M$ the centralizer of $A$ in $K$. Since $A$ is a torus, its centralizer in $G$ is connected [10, p. 287], and in fact has Lie algebra $m+\mathfrak{a}[\mathbf{1 0}$, p. 263]. The centralizer must therefore be $M_{e} \cdot A\left[10\right.$, p. 271], where $M_{e}=\exp _{G} \mathfrak{m}$ is the identity component of $M$. Hence $M \cdot A=M_{e} \cdot A$ and $M=M_{e} \cdot(M \cap A)$. Note that since $M \cap A$ is abelian, compact and has Lie algebra $m \cap \mathfrak{a}=0$ it is a finite abelian group. Furthermore, if $\sigma$ is the involution of $(G, K)$ and $m \in M \cap A$, then $\sigma(m)=m$ because $m \in K$, and $\sigma(m)=m^{-1}$ because $m \in V$, hence $M \cap A$ consists of involutions. We summarise these well-known arguments in the following proposition.

(5.1) Proposition. The centraliser of $A$ (in $G$ ) is $M A$ and this equals $M_{e} A$, where $M_{e}$ is the identity component of $M$. Hence $M=M_{e} \cdot(M \cap A)$, where $M \cap A$ is a finite abelian group of involutions.

(5.2) Proposition. The irreducible representations of $M A$ are $\eta \tilde{\otimes} e^{i \psi}$, where $\psi \in a^{*}$ satisfies

$$
e^{i \psi(H)}=1 \quad \text { whenever } \exp _{G}(H)=e,
$$

where $\eta$ is an irreducible representation of $M_{e}$ such that $\eta(a)$ is multiplication by $e^{i \psi}(a)$ for each $a \in M_{e} \cap A$, and where $\eta \tilde{\otimes} e^{i \psi}$ is defined by

$$
\eta \tilde{\otimes} e^{i \psi}(m a)=e^{i \psi}(a) \eta(m) \quad \forall m \in M_{e}, a \in A .
$$


Proof. Since $A$ centralises $M A$, an irreducible representation restricted to $A$ must act as scalar multiplication by a character. Since, by Proposition $1, M A=M_{e} A$, its restriction to $M_{e}$ must also be irreducible. The characters of $A$ have the form

$$
\exp _{G}(H) \mapsto e^{i \psi(H)} \quad \forall H \in \mathfrak{a},
$$

where $\psi \in a^{*}$ satisfies

$$
e^{i \psi(H)}=1 \quad \text { whenever } \exp _{G}(H)=e .
$$

An irreducible representation of $M A$ therefore has the form stated in the proposition.

The realisations of the irreducible representations of $M_{e}$ and $G$ given by the Borel-Weil-Bott theorem are defined by choices of maximal tori and of positive root systems with respect to these. We want to make compatible such choices in the following sense.

(5.3) Definition. Let $T_{1}$ be a maximal torus of $M_{e}$. Then $T_{1} \equiv T_{1} A$ is a maximal torus of both $M A$ and $G$.

Let $\Phi^{+}$be a set of positive roots of $g_{c}$ with respect to $T$; whence $\alpha \in \mathfrak{L}^{*}$ is a root of $(\mathfrak{m}+a)_{c}$ if and only if it is a root of $\mathfrak{g}_{c}$ and $\mathfrak{g}_{\alpha} \subset \mathfrak{m}$. Define

$$
\begin{aligned}
& P_{-}=\left\{\alpha \in \Phi^{+} \mid g_{\alpha} \subset \mathrm{m}_{\mathrm{c}}\right\}, \\
& P_{+}=\left\{\alpha \in \Phi^{+} \mid \mathfrak{g}_{\alpha} \not \subset \mathrm{m}_{\mathrm{c}}\right\} .
\end{aligned}
$$

Then $P_{-}$is a positive root system for $M_{\mathbf{c}}$ with respect to $T_{1}$, in the sense that $\alpha_{\mid \mathrm{t}_{1}}$, for

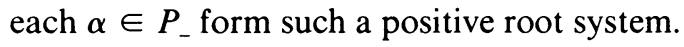

We say that $T_{1}$ and $T$ are compatible choices of maximal tori for $M_{e}$ and $M$ and that $\Phi^{+}$and $P_{-}$are compatible choices of positive root systems with respect to them.

In terms of compatibly chosen maximal tori and positive root systems we define the representation of $G$ holomorphically induced from the irreducible representations of $M A$.

(5.4) Definition. Let $\eta \tilde{\otimes} e^{i \psi}$ be an irreducible representation of $M A$.

Let $T_{1}$ and $T$ be compatible maximal tori of $M_{e}$ and $G$, and let $\Phi^{+}$be a system of positive roots of $G$ with respect to $T$, and let $P_{-}$and $P_{+}$be defined as in Definition 1.

Let $H_{\eta}$ be the representation space of $\eta$ defined by $T_{1}$ and $P_{1}$. Hence there is a unique character $\chi_{1}$ of $T_{1}$ such that $H_{\eta}$ is the space of smooth functions for $M_{e}$ satisfying

$$
\begin{gathered}
\chi\left(t_{1}\right) f\left(m t_{1}\right)=f(m) \quad \forall t_{1} \in T_{1}, m \in M_{e}, \\
X f=0 \quad \forall X \in n_{-},
\end{gathered}
$$

where

$$
n_{-} \equiv \sum_{\alpha \in P_{-}} \mathfrak{g}_{\alpha}
$$

On the space $\mathrm{C}^{\infty}\left(G, H_{\eta}\right)$ of smooth functions $F: G \rightarrow H_{\eta}$ we let $g_{\mathrm{c}}$ act as left invariant vector fields, and for each $X \in g_{\mathrm{c}}$ define $\nabla_{X}$ by

$$
\nabla_{X} F(g)(m) \equiv[\operatorname{Ad}(m) X] \cdot F(g)(m) \quad \forall m \in M_{e}, g \in G \text {. }
$$


Let

$$
n_{+} \equiv \sum_{\alpha \in P_{+}} g_{\alpha}
$$

The representation $\sigma_{\psi, \eta}$ of $G$, holomorphically induced from $\eta \tilde{\otimes} e^{i \psi}$, is the restriction of the left regular representation on $C^{\infty}\left(G, H_{\eta}\right)$ to the subspace of functions $F$ satisfying

$$
\begin{gathered}
\eta \tilde{\otimes} e^{i \psi}(m a) F(g m a)=F(g) \quad \forall g \in G, m \in M_{e}, a \in A, \\
\nabla_{X} F=0 \quad \forall X \in n_{+} .
\end{gathered}
$$

There is a natural identification between the representations $\sigma_{\psi, \eta}$ described above and those holomorphically induced from characters of $T$. For suppose that $\eta \tilde{\otimes} e^{i \psi}$ is an irreducible representation of $M A$ and $\eta$ is induced holomorphically from the character $\chi_{1}$ of $T_{1}$. Then $\chi_{1}$ and $e^{\mathrm{i} \psi}$ agree on $T_{1} \cap A$. Indeed, because $M_{e} \cap A$ is central in $M_{e}$, and $T_{1}$ is maximal abelian, $M_{e} \cap A=T_{1} \cap A$. Moreover $\eta(a)$ is required to act as multiplication by $e^{i \psi}(a)$ for each $a \in M_{e} \cap A$, but (xi) shows that it must act as multiplication by $\chi_{1}(a)$. Since $e^{i \psi}$ and $\chi_{1}$ agree on $T_{1} \cap A$, they define a character $\chi$ of $T=T_{1} A$ by

$$
\chi\left(t_{1} a\right)=\chi_{1}\left(t_{1}\right) e^{i \psi}(a) \quad \forall t_{1} \in T, a \in A .
$$

We then have

(5.5) Proposition. Let $\eta \tilde{\otimes} e^{i \psi}$ be an irreducible representation of MA. Let $T_{1}$ and $T$ be compatible maximal tori of $M_{e}$ and $G$, let $\Phi^{+}$be a system of positive roots of $G$ with respect to $T$, and let $P_{-}$and $P_{+}$be defined as in Definition 1 . Let $\chi_{1}$ be the character of $T_{1}$ from which $\eta$ is holomorphically induced, using $P_{-}$to define the holomorphy condition. Define the character $\chi$ of $T$ by

$$
\chi\left(t_{1} a\right)=\chi_{1}\left(t_{1}\right) e^{i \psi}(a) \quad \forall t_{1} \in T_{1}, a \in A .
$$

For each $F \in C^{\infty}\left(G, H_{\eta}\right)$ define $\hat{F}$ by

$$
\hat{F}: g \mapsto F(g)(e) \quad \forall g \in G
$$

and for each $f \in C^{\infty}(G)$ define $\breve{f}$ by

$$
\check{f}(\mathrm{~g}): m \mapsto f(\mathrm{gm}) \quad \forall m \in M_{e}, g \in G .
$$

Then $F \rightarrow \check{F}$ defines a morphism of representations $\sigma_{\psi, \eta} \mapsto \mathscr{H}_{\chi}$ whose inverse is $f \mapsto \check{f}$.

It follows that each $\sigma_{\psi, \eta}$ is either irreducible or zero, and that each irreducible representation of $G$ occurs in this form for a unique choice of $\eta$ and $\psi$.

In order to formulate an analogue of Theorem 1 for a compact Riemannian symmetric pair we require $K$-invariant functions to play the role of $s_{\psi}$ (see $\S 4$ ). We require these to belong to the representation spaces of certain of the representations $\sigma_{\psi, \eta}$. An irreducible representation of $G$ containing a $K$-invariant vector is called a $K$-class 1 representation. In respect of these we have

(5.6) TheOREm (CARTAN [3], SEE ALSo [15]). An irreducible representation $\sigma_{\psi, \eta}$ of $G$ is $K$-class 1 if and only if $\eta=1$, the trivial representation of $M$. The subspace of $K$-invariant vectors is one-dimensional. 
As to the existence of irreducible representations of the form $\sigma_{\psi, 1}$, we have the following well-known result.

(5.7) Proposition. There is a basis $\phi_{1}, \ldots, \phi_{l}$ of $a^{*}$ for which $\sigma_{\phi_{1}, 1}, \ldots, \sigma_{\phi_{1}, 1}$ are nonzero (hence irreducible) representations of $G$.

Proof. If $\alpha$ is a root of $g_{\mathrm{c}}$ with respect to $T$, let $\bar{\alpha}$ be its restriction to a. Note that $e^{i \bar{\alpha}}$ is the restriction to $A$ of the character $e^{i \alpha}$ of $T$. Recall that $\bar{\alpha}$ is called a restricted root, is called positive if $\alpha \in P_{+}$and simple if it is positive and not the sum of two other positive restricted roots. If $\beta_{1}, \ldots, \beta_{l}$ is the set of all simple restricted roots, then they are a basis for a $\left[10\right.$, p. 292]. We define $\phi_{i}=2 \beta_{i}$ for $i=1, \ldots, l$. By the remark above, for each $i=1, \ldots, l, e^{i \phi_{i}}$ is a well-defined character of $A$, and since $e^{i \phi_{1}}=\left(e^{i \beta_{l}}\right)^{2}$ and $M \cap A$ consists of involutions, it agrees with the trivial representation 1 of $M$ on $M \cap A$. Hence $\sigma_{\phi_{1}, 1}$ is well defined.

We claim that, for each $i=1, \ldots, l, \sigma_{\phi_{i}, 1}$ is nonzero. Indeed, $\sigma_{\phi_{i}, 1}=\mathscr{H}_{\chi_{i}}$, where $\chi_{i}$ is the character of $T$ whose restriction to $T_{1}$ is 1 and to $A$ is $e^{i \phi_{i}}$. Hence $-i d \chi_{i e}$ vanishes on $\mathrm{t}_{1}$ and equals $\phi_{i}$ on $a$. If $\beta_{i}=\bar{\alpha}_{i}$ and $\alpha_{i}$ is represented by $t_{\alpha_{i}}$ under the Killing form, it follows easily that $i d \chi_{i e}=\left(a_{\alpha_{i}},-\right)$, where $t_{\alpha_{i}}=t_{\alpha_{i}}^{\prime}+a_{\alpha_{i}}$ with $t_{\alpha_{i}}^{\prime} \in \mathrm{t}_{1}$, $a_{\alpha_{i}} \in$ a. For any positive root $\alpha$, represented by $t_{\alpha}$ under the Killing form, we then have $[\mathbf{1 0}$, p. 291]

$$
-i d \chi_{i e}\left(t_{\alpha}\right)=\left(a_{\alpha_{t}}, a_{\alpha}\right) \leq 0,
$$

where $t_{\alpha}=t_{\alpha}^{\prime}+a_{\alpha}, t_{\alpha} \in \mathrm{t}_{1}, a_{\alpha} \in \mathfrak{a}$. Hence $\mathscr{H}_{\chi_{1}}$, and thus $\sigma_{\phi_{i}, 1}$, is nonzero.

From now on, we fix the basis $\phi_{1}, \ldots, \phi_{l}$ of $a^{*}$ as in Proposition 5.7 above.

(5.8) Definition. For each $i=1, \ldots, l$ let $s_{i}$ be the unique $K$-invariant vector of $\sigma_{\phi_{1}, 1}$ whose value on $K$ is 1 .

(5.9) Lemma. For each $k=1, \ldots$, l and $v \in V$

$$
\left|s_{k}\left(\exp _{G}-t v\right)-e^{i t \phi_{k}(v)}\right|=O\left(t^{2}\right)
$$

Proof. Regarding each $v \in V$ as a tangent vector to $G$ at $e$, the result follows by Taylor's theorem, once we prove

$$
v \cdot s_{k}=i \phi_{k}(v) \quad \forall v \in V .
$$

Let $V=\mathfrak{a}+q_{0}$, where $q_{0}$ is the orthogonal complement of a under the Killing form, and let $q=q_{0} \otimes$ C. By [10, p. 288], $q$ is spanned by $X_{\alpha}-\theta X_{\alpha}, \alpha \in P_{+}$, where $X_{\alpha} \in g_{\alpha}$. Since $X_{\alpha}+\theta X_{\alpha}$ lies in $\mathfrak{f}_{\mathbf{c}}$, and $s_{k}$ is $K$-invariant

$$
\left(X_{\alpha}+\theta X_{\alpha}\right) \cdot s_{k}=0 \quad \forall \alpha \in P_{+} .
$$

By the holomorphy condition defining $\sigma_{\psi_{k} .1}$, and since $\nabla_{X} s(e)=X s(e)$, we have $X_{\alpha} \cdot s=0 \forall \alpha \in P_{+}$. Hence

$$
v \cdot s_{k}=0=i \phi_{k}(v) \quad \forall v \in q_{0} .
$$

If $v \in \mathfrak{a}$, then by the induced representation condition

$$
s_{k}\left(\exp _{G}-t v\right)=e^{i \phi_{k}(v)} s(e)=e^{i \phi_{k}(v)}
$$

so that $v \cdot s_{k}=i \phi_{k}(v)$. This completes the proof. 
(5.10) Definition. For each $c=\left(c_{1}, \ldots, c_{l}\right) \in \mathbf{N}^{\prime}$ define $s^{c} \equiv s_{1}^{c_{1}} \cdot s_{2}^{c_{2}} \cdots s_{l}^{c_{l}}$.

(5.11) Lemma. Let $\psi=\gamma_{1} \phi_{1}+\cdots+\gamma_{l} \phi_{l}$ with $\gamma=\left(\gamma_{1}, \ldots, \gamma_{l}\right) \in \mathbf{R}^{l}$. Let $\left\{c_{n}\right\}_{1}^{\infty}$ be a sequence in $\mathbf{N}^{\prime}$ such that

$$
c_{k n} / n=\gamma_{k}+O(1 / n) \text { where } c_{n}=\left(c_{1 n}, \ldots, c_{l n}\right) .
$$

Then $\left|s^{c_{n}}\left(\exp _{G} v / n\right)-e^{i \psi(v)}\right|=O(1 / n)$.

Proof. By Lemma 5.9

$$
\left|\ln s_{k}\left(\exp _{G}-v / n\right)-i \phi_{k}(v)\right|=O\left(1 / n^{2}\right)
$$

and so, since $c_{k n}=O(n)$

$$
\left|c_{k n} \ln s_{k}\left(\exp _{G}-v / n\right)-i c_{k n} \phi_{k}(v) / n\right|=O(1 / n)
$$

and

$$
\left|c_{k n} \ln s_{k}\left(\exp _{G}-v / n\right)-i \gamma_{k} \phi_{k}(v)\right|=O(1 / n) .
$$

Adding these for $k=1, \ldots, l$ gives

$$
\left|\ln s^{c_{n}}\left(\exp _{G}-v / n\right)-i \psi(v)\right|=O(1 / n)
$$

from which the lemma follows.

We now proceed to formulate the theorem describing how each generic irreducible representation $\rho_{\psi, \eta}$ of $V \rtimes K$ is approximated by a sequence of irreducible representations of $G$. First note that if $\eta$ is an irreducible representation of $M$, then, as observed above, its restriction to $M \cap A$ acts as multiplication by a character of $M \cap A$. This character can be extended to a character of $A$. Indeed, since $M \cap A$ consists of involutions it is easy to describe such extensions explicitly. Hence, given $\eta$, there exists a character $e^{i \psi_{0}}$ of $A$ such that $\sigma_{\psi_{0}, \eta}$ is defined (although it may be zero). Secondly, by the definition of $\phi_{1}, \ldots, \phi_{l}$ and [10, p. 323], every coadjoint orbit of $K$ in $V^{*}$ not only passes through $a^{*}$, but contains a $\psi$ such that $\psi=\gamma_{1} \phi_{1}+$ $\cdots+\gamma_{1} \phi_{l}$ with $\gamma_{k} \geq 0, k=1, \ldots, l$. Hence, for each irreducible representation $\rho_{\psi, \eta}$ of $V \rtimes K$ we may assume $\psi$ to have this form. Moreover, $\rho_{\psi, \eta}$ is generic if and only if $\gamma_{k}>0, k=1, \ldots, l$.

(5.12) THEOREM 2. Let $\rho_{\psi, \eta}$ be a generic irreducible representation of $V \rtimes K$, where $\psi=\gamma_{1} \phi_{1}+\cdots+\gamma_{l} \phi_{l}$ with $\gamma_{k}>0, k=1, \ldots$, l. Let $\left\{c_{n}\right\}_{1}^{\infty}$ be a sequence of l-tuples of nonnegative integers such that

$$
c_{n k} / n=\gamma_{k}+O(1 / n), \quad k=1, \ldots, l, \text { where } c_{n}=\left(c_{n 1}, \ldots, c_{n l}\right) .
$$

Let $\psi_{0} \in a^{*}$ be such that $\sigma_{\psi_{0}, \eta}$ is defined and let $\psi_{n}=\psi_{0}+c_{n 1} \phi_{1}+\cdots+c_{n l} \phi_{l}$. Let $\mathscr{H}_{n}$ be the representation space of $\sigma_{\psi_{n}, \eta}$. Then,

(i) $\mathscr{H}_{n} \neq 0$ for large enough $n$.

(ii) For each $p \in \mathbf{N}, n \geq p$ and $f \in \mathscr{H}_{p}, s^{c_{n}-c_{p}} \cdot f \in \mathscr{H}_{n}$. Define, for $f \in \mathscr{H}_{p}$

$$
\bar{\sigma}_{\psi_{n}, \eta}(v k)(f) \equiv \sigma_{\psi_{n}, \eta}\left(\pi_{1 / n}(v k)\right)\left(s^{c_{n}-c_{p}} f\right)
$$


Then

(iii) $\left|\bar{\sigma}_{\psi_{,}, \eta}(v k)(f)\left(k_{0}\right)-\rho_{\psi, \eta}(v k)(f)\left(k_{0}\right)\right|=O(1 / n)$ and hence

$$
\left\|\bar{\sigma}_{\psi_{n}, \eta}(v k)(f)\left(k_{0}\right)-\rho_{\psi, \eta}(v k)(f)\left(k_{0}\right)\right\|=O(1 / n)
$$

in $L^{2}\left(K, H_{\eta}\right)$.

(iv) If $\mathscr{H}_{n \mid K}$ denotes the functions which are restrictions to $K$ of those in $\mathscr{H}_{n}$, then $\mathscr{H}_{\left.n\right|_{k}} \subset \mathscr{H}_{n+\left.1\right|_{K}}$ and $\bigcup_{n=1}^{\infty} \mathscr{H}_{\left.n\right|_{K}}$ is dense in $H_{\psi, \eta}$, the representation space of $\rho_{\psi, \eta}$.

Proof. (i) Let $\eta$ be holomorphically induced from $\chi_{1}$. Then $\sigma_{\psi_{n}, \eta}=\mathscr{H}_{\chi}$, where $\chi$ is defined on $T=T_{1} A$ by

$$
\chi\left(t_{1} a\right)=\chi_{1}\left(t_{1}\right) e^{i \psi_{n}}(a) \quad \forall t_{1} \in T, a \in A .
$$

Now for any positive root $\alpha$ of $g_{c}$ with respect to $T$, $-i d \chi_{e}\left(t_{\alpha}\right)=-i d \chi_{1 e}\left(t_{\alpha}^{\prime}\right)+\psi_{n}\left(a_{\alpha}\right)$, where $t_{\alpha} \in \mathrm{t}$ represents $\alpha$ under the Killing form, and $t_{\alpha}=t_{\alpha}^{\prime}+a_{\alpha}$ with $t_{\alpha}^{\prime} \in \mathrm{t}_{1}$ and $a_{\alpha} \in a$. If $\alpha \in P_{-}$, then $a_{\alpha}=0$ and $-i d \chi_{1 e}\left(t_{\alpha}^{\prime}\right) \leq 0$ because $\eta$ is nontrivial. If $\alpha \in P_{+}$, then, by definition of $\phi_{k}, \phi_{k}\left(a_{\alpha}\right) \leq 0, k=1, \ldots, l$, and not all of these are zero. Hence, because $\gamma_{k}>0, k=1, \ldots, l, \psi_{n}\left(a_{\alpha}\right) \rightarrow-\infty$ as $n \rightarrow \infty$, and $-i d \chi_{e}\left(t_{\alpha}\right)$ $<0$ for $n$ large enough. It follows that $\sigma_{\psi_{n}, \eta}$ is nonzero for $n$ large enough.

(ii) Let $f \in \mathscr{H}_{p}$ and consider $s_{k} \cdot f$. Note that by the definition of $\sigma_{\phi_{k}, 1}, s_{k}$ is a function $G \rightarrow \mathbf{C}$ so that the product makes sense. We show that $s_{k} f$ belongs to the representation space of $\sigma_{\psi_{p}+\phi_{k}, \eta}$. Repeated application of this result for $k=1, \ldots, l$ gives (ii). Now $s_{k} f, G \rightarrow H_{\eta}$, and for $m a \in M A$ and $g \in G$

$$
\begin{aligned}
e^{i\left(\psi_{p}+\phi_{k}\right)}(a) \eta(m) s_{k} f(g m a) & =e^{i \phi_{k}}(a) s(g m a) e^{i \psi_{p}}(a) \eta(m) f(g m a) \\
& =s_{k}(g) f(g)=s_{k} f(g) .
\end{aligned}
$$

Moreover, by the holomorphy condition in Definition 5.4

$$
\nabla_{X} s_{k} f=\nabla_{X} s_{k} \cdot f+s_{k} \cdot \nabla_{X} f=0 \quad \forall X \in \mathfrak{n}_{+} .
$$

Hence $s_{k} f \in \sigma_{\psi_{p}+\phi_{k}, \eta}$.

(iii)

$$
\begin{aligned}
& \bar{\sigma}_{\psi_{n}, \eta}(v k)(f)\left(k_{0}\right)=\left(s^{c_{n}-c_{p}} \cdot f\right)\left(k^{-1} \exp _{G}\left(-\frac{v}{n}\right) k_{0}\right) \\
& =s^{c_{n}-c_{p}}\left(k^{-1} k_{0} \exp _{G}\left(-\operatorname{Ad}\left(k_{0}^{-1}\right) \frac{v}{n}\right)\right) f\left(k^{-1} k_{o} \exp _{G}\left(-\operatorname{Ad}\left(k_{0}^{-1}\right) \frac{v}{n}\right)\right) \\
& =s^{c_{n}-c_{p}}\left(\exp _{G}\left(-\operatorname{Ad}\left(k_{0}^{-1}\right) \frac{v}{n}\right)\right) f\left(k^{-1} k_{0} \exp _{G}\left(-\operatorname{Ad}\left(k_{0}^{-1}\right) \frac{v}{n}\right)\right) \text {. }
\end{aligned}
$$

Now

$$
\left|s^{c_{n}-c_{p}}\left(\exp _{G}\left(-\operatorname{Ad}\left(k_{0}^{-1}\right) \frac{v}{n}\right)\right)-e^{i \psi\left(\operatorname{Ad}\left(k_{0}^{-1}\right) v\right)}\right|=O\left(\frac{1}{n}\right)
$$

by Lemma 5.11, and

$$
\left|f\left(k^{-1} k_{0} \exp _{G}\left(-\operatorname{Ad}\left(k_{0}^{-1}\right) \frac{v}{n}\right)\right)-f\left(k^{-1} k_{0}\right)\right|=O\left(\frac{v}{n}\right)
$$

by the mean value theorem. Therefore we have

$$
\left|\bar{\sigma}_{\psi_{n}, \eta}(v k)(f)\left(k_{0}\right)-\rho_{\psi, \eta}(v)\left(f_{\mid K}\right)\left(k_{0}\right)\right|=O(1 / n) .
$$


Since $s^{c_{n}-c_{p}}$ and $f$ are real analytic, the $O(1 / n)$ estimates are uniform on $K$. Hence by integration over $K$, we obtain

$$
\left\|\sigma_{\psi_{n}, \eta}(v k)(f)_{\mid K}-\rho_{\psi, \eta}(v k)\left(f_{\left.\right|_{K}}\right)\right\|=O(1 / n) .
$$

(iv) Since $s^{c_{n}-c_{p}}$ has the value 1 on $K, s^{c_{n}-c_{p}} f$ has the same restriction to $K$ as $f$ for each $f \in \mathscr{H}_{p}$. Hence for every $n \geq p, \mathscr{H}_{\left.p\right|_{K}} \subseteq \mathscr{H}_{\left.n\right|_{K}}$. From (iii) it follows that for each $v k \in V \rtimes K$ and $f \in \mathscr{H}_{p}, \rho_{\psi, \eta}(v k)\left(f_{\left.\right|_{K}}\right)$ lies in the $L^{2}\left(K, H_{\eta}\right)$ closure of $\bigcup_{n=1}^{\infty} \mathscr{H}_{\left.n\right|_{K}}$. By (i) this is nonzero, and so carries a nonzero subrepresentation of $\rho_{\psi, \eta}$. Since $\rho_{\psi, \eta}$ is irreducible, the closure in $L^{2}\left(K, H_{\eta}\right)$ of $\bigcup_{n=1}^{\infty} \mathscr{H}_{\left.n\right|_{K}}$ must be $H_{\psi, \eta}$.

6. The nongeneric case. Recall that the irreducible unitary representations of the Cartan motion group $V \rtimes K$ have the form $\rho_{\psi, \eta}$ (see $\$ 3$ ), where $\psi \in a^{*}$ and $\eta$ is an irreducible unitary representation of $K_{\psi}$, the stabilizer of $\psi$ under the coadjoint action of $K$ on $V$. Generically $K_{\psi}=M$, the stabilizer of a under the adjoint action. But certainly one can have $K_{\psi} \supsetneqq M$. We call this the nongeneric case and we discuss the corresponding extensions of Theorems 1 and 2, with certain exceptions in respect of Theorem 2. Note that nongeneric refers to the representations of $V \rtimes K$ and not necessarily to the approximating ones from $G$.

Fix a maximal torus $T_{1}$ of $K_{\psi}$ with Lie algebra $\mathrm{t}_{1}$ and let $a_{1}$ be the centralizer of $\mathrm{t}_{1}$ in $a$. Then $\mathfrak{h}_{c}=\left(t_{1}+a\right) \otimes c$ is a Cartan subalgebra of $g_{c}$ and we let $\Phi$ be the set of roots of $g_{c}$ with respect to $\mathfrak{h}_{\mathbf{c}}$. The centralizer of $a_{1 \mathbf{c}}$ in $g_{c}$ is

$$
\mathfrak{m}_{1 \mathbf{c}}=\sum_{\alpha \in \Phi, \alpha\left(a_{1}\right)=0} \mathfrak{g}_{\alpha} \text {. }
$$

If $A_{1}=\exp _{G} a_{1}$ one can show that the centraliser of $A_{1}$ in $G$ has the form $M_{1} A_{1}$, where $M_{1}$ is a reductive $\theta$-stable subgroup of $G$ with Lie algebra $\mathrm{m}_{1}$, the real part of $\mathfrak{m}_{1 \mathbf{c}}$ (under the conjugation of $\mathfrak{g}_{\mathbf{c}}$ with respect to $\mathfrak{g}$ ).

If $(G, K)$ is a Riemannian symmetric pair of noncompact type, then [24] $M_{1} \cap A_{1}$ $=e$, and $K_{\psi}=M_{1} \cap K$ is a maximal compact subgroup of $M_{1}$. Choosing a set $\Phi^{+}$of positive roots of $\mathfrak{g}_{\mathbf{c}}$ with respect to $\mathfrak{h}_{\mathbf{c}}$ we define, as in $\S 4$

$$
\begin{gathered}
P_{-}=\left\{\alpha \in \Phi^{+} \mid \alpha(H)=O \forall H \in a_{1}\right\}, \\
P_{+}=\left\{\alpha \in \Phi^{+} \mid \alpha \notin P_{-}\right\}, \\
\mathfrak{n}_{+}=\sum_{\alpha \in P_{+}} g_{\alpha} .
\end{gathered}
$$

Let $\mathfrak{n}$ be the real part of $\mathrm{n}_{\mathrm{c}}$ and $N=\exp _{G} \mathfrak{n}$. Then $P=M_{1} A_{1} N$ is a parabolic subgroup of $G$, and we define an irreducible representation $\eta^{\prime} \otimes e^{\gamma}$ of $P$ by

$$
\eta^{\prime} \otimes e^{\gamma}(\text { man })=e^{\gamma}(a) \eta^{\prime}(m) \quad \forall \text { man } \in M_{1} A_{1} N,
$$

where $\eta^{\prime}$ is a discrete series representation of $M_{1}, \gamma \in a_{1}^{*}$ and $e^{\gamma}$ is the map $a \mapsto e^{\gamma(\log a)}$. Let $H$ be the Cartan subgroup which is the stabiliser of $\mathfrak{h}_{c}$ under the adjoint action of $G$. An $H$-series representation of $G$ is, by definition, one induced from a representation $\eta \otimes e^{\gamma}$ of $P$. As such, it is realised on a space of functions $f$ : $G \rightarrow H_{\eta}$ satisfying

$$
\eta^{\prime} \otimes e^{\gamma}(p) f(g p)=f(g) \quad \forall g \in G, p \in P .
$$


An $H$-series representation gives rise to a unitary representation if and only if $\gamma=\mu$ $+i \phi$ with $\phi \in \mathfrak{a}_{1}^{*}$ and $\mu$ a particular linear map in $a^{*}$. In this case, one can take for the representation space the functions satisfying 6.4 and

$$
\int_{K}\|f(k)\|^{2} d \mu<\infty
$$

We denote this $H$-series representation by $\sigma_{\phi, \eta^{\prime}}$ and its representation space by $\mathscr{H}_{\phi, \eta^{\prime}}$.

Given an irreducible representation $\eta$ of $K_{\psi}$ we choose a discrete series representation $\eta^{\prime}$ of $M_{1}$ whose restriction to $K_{\psi}$ contains $\eta$, or more correctly, has $\eta$ as a quotient. This can always be done. One can, for example, note that discrete series representations are spinor induced from the irreducible representations of a maximal compact subgroup [1] and apply a form of Frobenius reciprocity. Alternatively one can choose the discrete series representation of $M_{1}$ whose highest weight is the same as that of $\eta$, and apply the formula of Blattner-Hecht-Schmidt to prove containment [8]. These give a natural choice of $\eta^{\prime}$. In general there will be many choices, as exemplified in the simplest case with $G=S L(2, \mathbf{R}), K=S O(2)$. Let $Q$ denote a projection of $\eta_{\mid K}^{\prime}$ onto $\eta$. Let

As in $\S 4$, define $s_{\psi}\left(k \exp _{G} v\right)=e^{-i \psi(v)}$. Then $s_{\psi} f \in \mathscr{H}_{(n+1) \psi, \eta^{\prime}}$ for any $f \in \mathscr{H}_{n \psi, \eta^{\prime}}$.

$$
(\Psi f)(k) \equiv Q(f(k))
$$

The map $\Psi$ generalises the mere restriction of functions to $K$ in the generic case. We have

$$
\Psi(f) \in H_{(n+1) \psi, \eta} \text { and } \Psi\left(s_{\Psi} f\right)=\Psi(f) .
$$

One can now follow the proof of Theorem 1 to obtain the following generalisation to the nongeneric case.

THEOREM 1'. With the notation above, define

$$
\overline{\boldsymbol{\sigma}}_{n \psi, \eta^{\prime}}(v k): f \mapsto \sigma_{n \psi, \eta^{\prime}}\left[\pi_{1 / n}(v k)\right]\left(s_{\psi}^{n} f\right)
$$

$\forall v k \in V \rtimes K$ and $f \in \mathscr{H}_{0, \eta^{\prime}}$.Then

$$
\operatorname{Lim}_{n \rightarrow \infty} \Psi\left[\bar{\sigma}_{n \psi}(v k) f\right]=\rho_{\psi \cdot \eta}(\Psi f) .
$$

Moreover, $\Psi\left(\mathscr{H}_{n \psi, \eta^{\prime}}\right)=H_{\psi, \eta} \forall n \in \mathbf{N}$ and the limit above is in the sense of $L^{2}\left(K, H_{\eta}\right)$.

Suppose now that $(G, K)$ is a Riemannian symmetric pair of the compact type. In this case we note that $K_{\psi} A_{1}$ is a closed subgroup of maximal rank, and we should like to use it as a replacement for $M A$ in the generic case. In contrast to this case, $K_{\psi} A_{1}$ is in general disconnected, and this causes difficulties in carrying through the arguments of $\S 5$. Using the root system $P_{+}$defined by (iii) above, one can define holomorphic induction from irreducible representations of $K_{\psi} A_{1}$. Since $A_{1}$ centralises $K_{\psi}$, such an irreducible representation has the form $\eta \otimes e^{i \phi}$, where $\eta$ is an irreducible representation of $K_{\psi}$ and $e^{i \phi}$ is a character of $A_{1}$. If $\sigma_{\phi, \eta}$ denotes the representation of $G$ holomorphically induced from $\eta \otimes e^{i \phi}$, one can show by the Cartan Theorem that there is a basis $\phi_{1}, \ldots, \phi_{m}$ of $a_{1}^{*}$ for which $\sigma_{\phi_{1}, 1}, i=1, \ldots, m$, are 
irreducible $K$-class 1 representations of $G$. One now arrives at the problem that, for an arbitrary $\eta$, it is not clear that one can find a character $e^{i \phi_{0}}$ of $A_{1}$ for which $\sigma_{\phi_{o}, \eta}$ is nonzero. One cannot guarantee, therefore, that the sequence of representations $\sigma_{\phi_{n}, \eta}$, which would be defined by analogy with Theorem 2 , is nontrivial.

This difficulty is circumvented in the generic case because $M A=M_{e} A$, and an irreducible representation of $M A$ is uniquely determined by one of $M_{e}$ and a character of $A$. The arguments leading to Theorem 2 can be followed almost verbatim in the nongeneric case, where, likewise, an irreducible representation of $K_{\psi} A_{1}$ is determined by one of $K_{\psi e}$ and a character of $A_{1}$. In this case one can relate representations holomorphically induced from $K_{\psi} A_{1}$ to those holomorphically induced from the connected subgroup $K_{\psi e} A_{1}$.

We can show that $K_{\psi}=K_{\psi e} \cdot(K \cap A)$, and that, for a suitable subgroup $F \subset K$ $\cap A, K_{\psi}=K_{\psi e} \rtimes F$. By Mackey's semidirect product analysis an irreducible representation $\eta$ of $K_{\psi}$ is determined by an irreducible representation $\bar{\eta}$ of $K_{\psi e}$ and a character of the subgroup $F$ whose elements $a$ stabilise $\bar{\eta}$ in the sense that $k \mapsto$ $\bar{\eta}\left(a k a^{-1}\right)$ is equivalent to $\bar{\eta}$. If this subgroup of $F$ lies in $A_{1}$, then we may extend the character of the subgroup to all of $A_{1}$ to obtain a character $e^{i \phi_{0}}$ say, and show from this that the sequence $\sigma_{\phi_{n}, \eta}$ is nontrivial. It is only in this case that we can prove the nongeneric analogue of Theorem 2.

\section{REFERENCES}

1. M. F. Atiyah and W. Schmid, A geometric construction of the discrete series for semisimple Lie groups, Invent. Math. 42 (1977), 1-62.

2. L. Auslander and B. Kostant, Polarisation and unitary representations of solvable Lie groups, Invent. Math. 14 (1971), 255-354.

3. E. Cartan, Sur la détermination d'un système orthogonal complet dans un espace de Riemann slmmetrique clos., Rend. Circ. Math. Palermo 53 (1929), 217-252.

4. J.-L. Clerc, Une formule asymptotique du type Mehler-Heine pour les zonales d' un espace riemannien symétrique, Studia Math. 57 (1976), 27-32.

5. A. H. Dooley and J. W. Rice, Contractions of rotation groups and their representations, Math. Proc. Cambridge Philos. Soc. 94 (1983), 509-517.

6. R. Gilmore, Lie groups, Lie algebras, and some of their applications, Wiley, New York, 1974.

7. V. Guillemin and S. Sternberg, Geometric asymptotics, Math. Surveys, no. 14, Amer. Math. Soc., Providence, R. I., 1977.

8. H. Hecht and W. Schmid, A proof of Blattner's conjecture, Invent. Math. 31 (1975), 129-154.

9. S. Helgason, Differential geometry and symmetric spaces, Academic Press, New York, 1962.

10. __ Differential geometry, Lie groups, and symmetric spaces, Academic Press, New York, 1978.

11. $\quad$ A duality for symmetric spaces with applications to group representations. III. Tangent space analysis, Adv. in Math. 36 (1980), 297-323.

12. J. E. Humphreys, Introduction to Lie algebras and representation theory, Springer-Verlag, New York, 1980.

13. E. Inönu and E. P. Wigner, On the contraction of groups and their representations, Proc. Nat. Acad. Sci. U.S.A. 39 (1953), 510-524.

14. A. Kleppner and R. L. Lipsman, The Plancherel formula for group extensions, Ann. Sci. Ecole Norm. Sup. (4) 5 (1972), 459-516.

15. M. Lasalle, Série de Laurent des fonctions holomorphes dans la complexification d'un espace symmetrique compacte, Ann. Sci. Ecole Norm. Sup. (4) 11 (1978), 167-210.

16. M. Lévy-Nahas, Deformation and contraction of Lie algebras, J. Math. Phys. 8 (1967), 1211-1222.

17. R. Lipsman, Orbit theory and harmonic analysis on Lie groups with co-compact nilradical, J. Math. Pures. Appl. 59 (1980), 337-374. 
18. G. W. Mackey, On the analogv between semisimple Lie groups and certain related semi-direct product groups, in Lie Groups and Their Representations (ed., I. M. Gelfand), Hilger, London, 1975.

19. Induced representations of groups and quantum mechanics, Benjamin, New York, 1968.

20. N. Mukunda, Expansion of Lie groups and representations of $S L(3, C)$, J. Math. Phys. 10 (1969), 897-911.

21. A. Nijenhuis and R. W. Richardson, Deformations of homorphisms of Lie groups and Lie algebras, Bull. Amer. Math. Soc. 73 (1967), 175-179.

22. E. J. Saletan, Contraction of Lie groups, J. Math. Phys. 2 (1961), 1-21.

23. G. Warner, Harmonic analysis on semi-simple Lie groups. I, Springer-Verlag, New York, 1972.

24. N. Wallach, Harmonic analysis on homogeneous spaces, Marcel Dekker, New York, 1973.

25. J. A. Wolf, Orbit method and non-degenerate series, Hiroshima Math J. 4 (1974), 619-628.

School of Mathematics, University of New South Wales, Kensington, N. S. W. 2033, Australia

School of Mathematical Sciences, Flinders University, Bedford Park, S. A. 5042, Australia 